\title{
CORE AND HALO PARTICLE DYNAMICS OF HIGH INTENSITY PROTON BEAMS
}

\author{
M. Pabst and K. Bongardt, Forschungszentrum Jülich GmbH, 52425 Jülich, Germany \\ A. P. Letchford, Rutherford Appleton Laboratory, Chilton, Didcot, UK
}

\section{Abstract}

A very critical design issue for a high intensity proton linac is to keep particle losses below $1 \mathrm{~W} / \mathrm{m}$ at the high energy end allowing unconstrained hands-on maintenance. For the ESS linac, with it's $214 \mathrm{~mA}$ peak current, Monte Carlo simulations with up to one million particles are performed. Single particle trajectories are shown along the linac demonstrating the existence of a space charge potential with non-linear terms and transverse-longitudinal coupling.

Radial halo particles at the final energy of $1.334 \mathrm{GeV}$ start at $70 \mathrm{MeV}$ at the boundary of the longitudinal phase space and also transversely close to a boundary of a 4D waterbag distribution. The single particle motion of these halo particles is characterised, on the average, by conservation of the sum of all three emittances. Trajectories are presented for both horizontal-vertical coupling and also transverse-longitudinal coupling. Core particles have a very different behaviour. For core particles, located around the rms emittances at $70 \mathrm{MeV}$, the single particle emittances can be either increased or decreased.

\section{INTRODUCTION}

High intensity proton linacs can be either pulsed $\mathrm{H}^{-}$- accelerators up to $5 \mathrm{MW}$ average beam power or cw $\mathrm{H}^{+}-$accelerators up to $130 \mathrm{MW}$ beam power. The major design problem is to reduce the particle loss along the linac down to $1 \mathrm{~W} / \mathrm{m}$ which corresponds to a loss rate below $10^{-7} / \mathrm{m}$. Losses above this limit prevent hands-on maintenance. Particle loss is caused by a small number of particles outside the dense beam core, called the beam halo. The origin and formation of the halo and it's dynamics are important issues for understanding the particle loss.

For this investigation of halo properties the underlying realistic high current linac design is the linac of the European Spallation Source (ESS) [1][2]. The main part of the $5 \mathrm{MW}$ ESS linac is a coupled cavity linac (CCL) at $700 \mathrm{MHz}$ with an effective bunch current of $214 \mathrm{~mA}$ which accelerates the beam from $70 \mathrm{MeV}$ up to 1.334 $\mathrm{GeV}$. The CCL is designed to have a tune depression of about 0.8 in all three planes. This non space charge dominated design results in less than $10 \%$ RMS emittance increase, preserves the cylindrical bunch shape and reduces the sensitivity to mismatch and tolerances [3]. At the end of the linac at $1.334 \mathrm{GeV}$, a beam halo can be recognised which contains less than $10^{-3}$ of the particles. To understand the properties and dynamics of these halo particles, single particle trajectories have been examined.

\section{PROPERTIES OF THE HALO}

Results from Monte Carlo simulations with 200000 particles are presented for the realistically designed ESS CCL. The output distribution of a preceding DTL was transferred into the CCL. No effect can be associated with the frequency jump and the change from a singlet to a doublet focussing system. Space charge calculations are fully three dimensional and no symmetries are assumed. At the input to the DTL at $5 \mathrm{MeV}$, the transverse and longitudinal phase spaces are filled independently with waterbag distributions. The space charge forces in such a distribution can be calculated analytically [4].
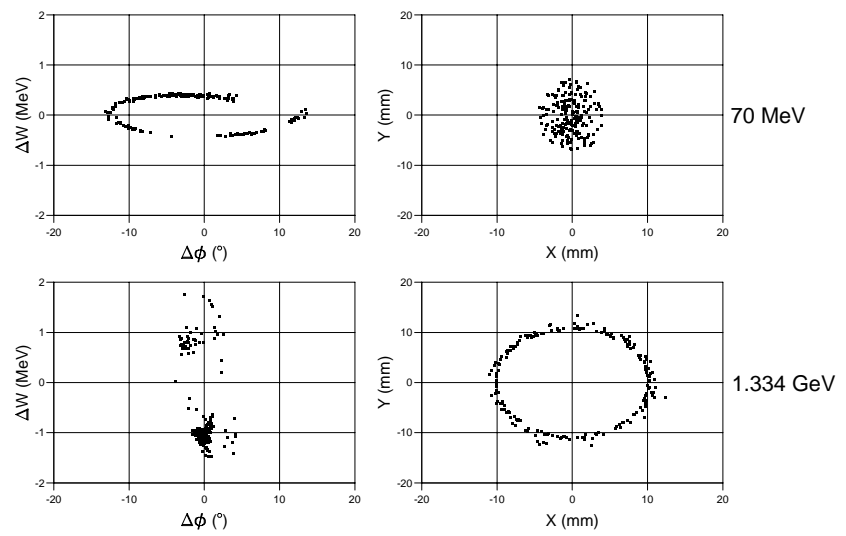

Figure 1: Longitudinal phase space $(\Delta \mathrm{W}, \Delta \phi)$ and real space $(\mathrm{X}, \mathrm{Y})$ projections at input $(70 \mathrm{MeV})$ and at output $(1.334 \mathrm{GeV})$ for the 200 outermost particles in $(\mathrm{X}, \mathrm{Y})$ space (halo particles) at the linac end.

In Figure 1, the 200 outermost radial halo particles are shown together with their position in the longitudinal phase space at $1.334 \mathrm{GeV}$. Also shown are the corresponding real space and longitudinal phase space positions at $70 \mathrm{MeV}$. There is obviously a strong transverselongitudinal coupling because all the radial halo particles at $1.334 \mathrm{GeV}$ start at the boundary of the longitudinal phase space at $70 \mathrm{MeV}$. Please note that the chosen 200 halo particles are not the outermost ones in real space at all energies. These particles are oscillating through the core. Also, they are not necessarily the outermost ones in either the horizontal or vertical phase space

In Figure 2, the same particles are shown in the transverse phase space at $70 \mathrm{MeV}$. All of the particles start from close to the boundary of the four dimensional transverse hyper-ellipsoid. The $\eta$ 's and the $\zeta$ 's corresponds to the standard circle transformation of single particle coordinates and velocities. In Figure $2 \mathrm{c}$ the absolute value 
of the transformed transverse velocity is plotted against the transformed transverse radius. Areas inside circles of Figure $2 \mathrm{c}$ are proportional to the sum of the transverse emittance. The inner line in Figure $2 c$ is the boundary for a 4D waterbag distribution (total emittance $=6 \mathrm{x} \mathrm{rms}$ emittance) with the same rms emittances as the beam. The dashed line corresponds to the boundary for the actual distribution where the total emittance $=7.68 \mathrm{x} \mathrm{rms} \mathrm{emittance}$.
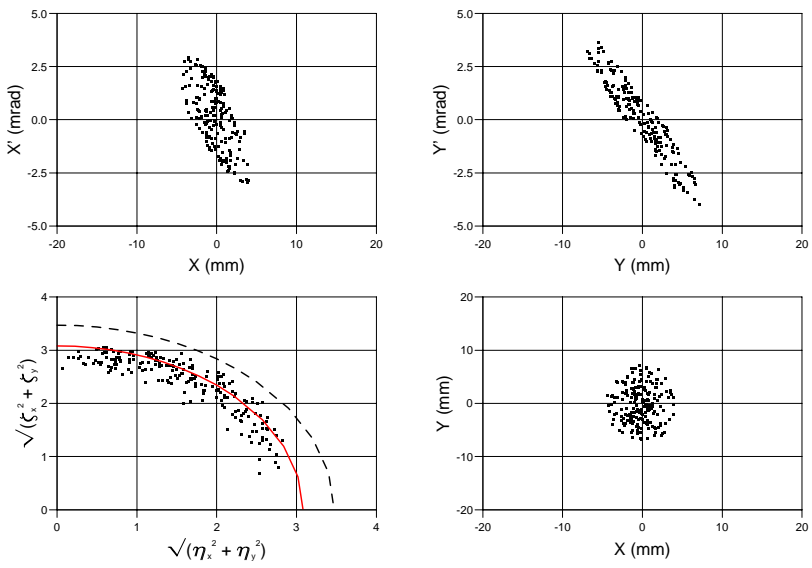

Figure 2: Transverse phase space projections of the halo particles at $70 \mathrm{MeV}$.

This strong horizontal-vertical correlation and the coupling to the longitudinal plane is also apparent at $1.334 \mathrm{GeV}$ as shown in Figure 3a. Here the sum of the normalised single particle emittances $\mathrm{Ex}+\mathrm{Ey}+\mathrm{Ez}$ is plotted against the sum of the normalised transverse emittances $\mathrm{Ex}+\mathrm{Ey}$, at $70 \mathrm{MeV}$ and $1.334 \mathrm{GeV}$ respectively, for the 200 radial halo particles. Evident is an increase in the sum of the transverse emittances, whereas the total sum stays constant on average, indicating a decrease in the longitudinal emittance on average. Figure 1 and Figure 3 are both a consequence of a transverse longitudinal coupling term in the space charge potential. As a result radial core particles at $70 \mathrm{MeV}$ become the outermost radial particles at the linac end. This process goes together with the exchange of emittances between the longitudinal and transverse directions.

For comparison, in Figure $3 \mathrm{~b}$ the same parameters are plotted for particles in the beam core at $70 \mathrm{MeV} .200$ particles are chosen which have single particle emittances in a range of $80 \%-120 \%$ of the rms emittance in all three planes at $70 \mathrm{MeV}$. These core particles show completely different behaviour than the particles which become the radial halo at $1.334 \mathrm{GeV}$. Their total transverse emittance as well as their longitudinal emittance can either increase or decrease. Note that these core particles would be located in the lower left corner of Figure $3 \mathrm{a}$.

Figures 4 and 5 show two typical halo particle single particle trajectories. Figure 4 depicts a particle with strong horizontal-vertical coupling. This leads to a rosette form in the radial plane as the elliptical path precesses. The longitudinal emittance is large but conserved in this case.

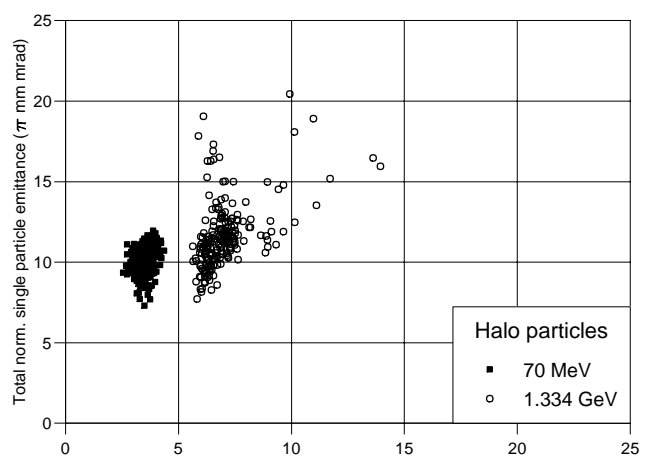

Sum of norm. transverse single particle emittances $(\pi \mathrm{mm} \mathrm{mrad})$

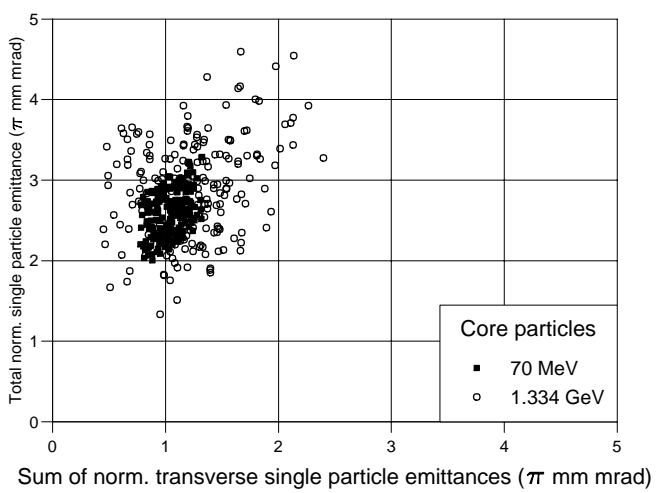

Figure 3: Plot of total normalized single particle emittance $(\mathrm{Ex}+\mathrm{Ey}+\mathrm{Ez})$ as a function of the transverse single particle emittance $(\mathrm{Ex}+\mathrm{Ey})$ for the halo particles (upper graph) and some 200 selected core particles (lower graph)

Figure 5 represents a longitudinal-vertical coupling. The horizontal emittance is much smaller than the vertical one here. The longitudinal emittance decreases as the vertical emittance increases, the sum remaining more or less constant. The rosette form in the $(\mathrm{Y}, \Delta \phi)$ plane is not as evident as in Figure 4. One reason may be the presence of non-linear external forces at the bunch head and tail. Also the difference in the transverse and longitudinal phase advance prevents a regular form, as in Figure 4, from appearing.

This analysis of halo particle properties is somewhat different to results obtained by the particle-core model [6]. Normally, an unbunched space charge dominated beam with tune depressions below 0.5 is considered The space charge forces are assumed to be linear inside the core. Test particles are positioned outside the core. Their motion, caused by strong mismatch, is studied. Chaotic behaviour can be seen. This model cannot describe how core particles can become halo particles. However, phase space boundaries can be obtained. 

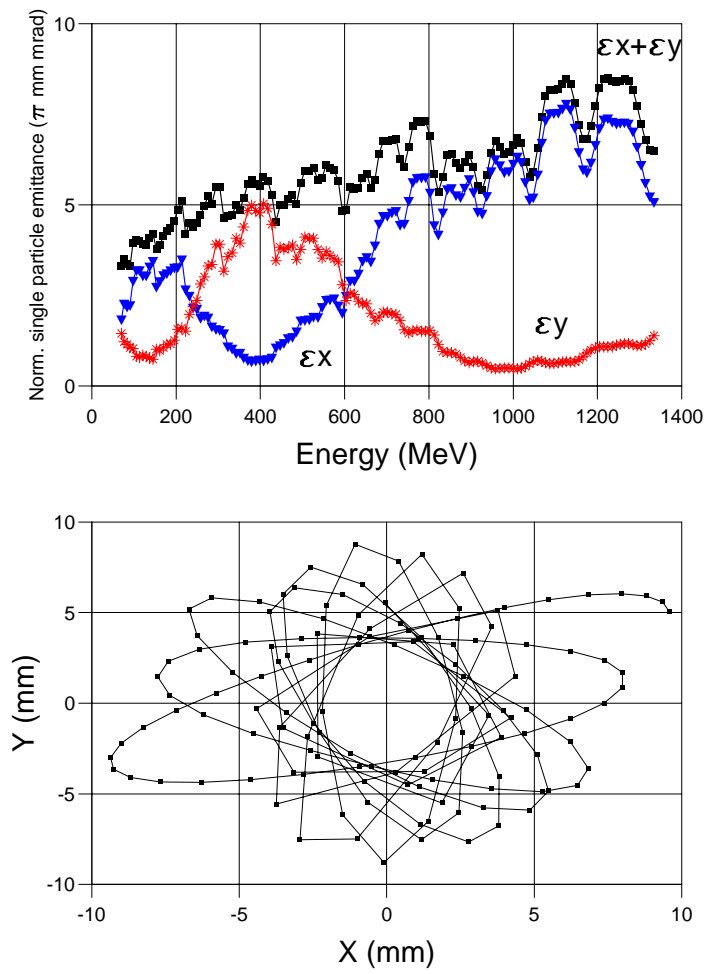

Figure 4: Single particle emittances and trajectories along the ESS CCL of a selected halo particle.

\section{HALO PRODUCTION RATE}

As has been recognised before from investigations of dc beams [5], halo production in a bunched beam is increased due to mismatch [3]. In the results presented above, there is about $60 \%$ change in the matched transverse beam radii, due mainly to the change in the number of accelerating cells per focusing period. In addition, the $20 \%$ longitudinal dc emittance growth which occurs in the preceding DTL has not been corrected for at the CCL input. By injecting a longitudinally matched beam, the longitudinal halo, which is not shown here, is substantially decreased. The driving force for the halo production is the non-linear space charge potential, associated with the non-self consistent distribution of a cylindrical bunch. Even for a perfectly rms matched beam there will be halo production. Mismatch, tolerances and errors then enhance the halo production rate.

This is somewhat different from the situation for a dc beam. Here, self consistent particle distributions are known which result in a zero halo production rate for a matched beam. Mismatch is then the driving term for halo production, not only an enhancement factor. More realistic unbunched distributions are associated with nonlinear space charge forces with an elliptical boundary in real space. It appears to be difficult to scale the halo production rate from a dc beam to a bunched beam as in the latter case the transverse-longitudinal coupling cannot be ignored.
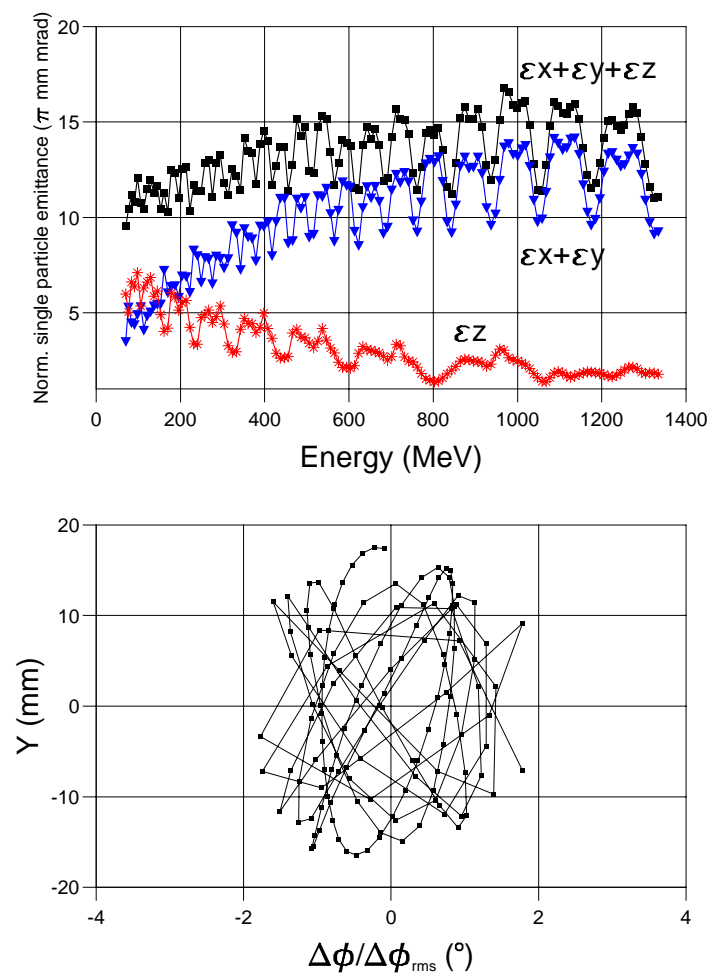

Figure 5: Single particle emittances and trajectories along the ESS CCL of another selected halo particle.

In order to estimate a particle loss rate at the beam pipe, Monte Carlo simulations with one million particles have been carried out for the first third of the CCL. From these on-going studies it is hoped to extrapolate, using data for 200000 and 1000000 particles, the particle distribution at the pipe radius. Quadrupole displacement errors have also been included in some simulations. As expected, even for beam displacements of up to $4 \mathrm{~mm}$, the particle distribution is only weakly effected. More dramatic changes are expected when sextupole errors are included.

\section{REFERENCES}

[1] ESS A Next Generation Neutron Source for Europe, Volume 3, The ESS Feasibility Study, March 1997.

[2] I. S. K. Gardner et al., these proceedings.

[3] M. Pabst et al., LINAC 96, Geneva, p27.

[4] M. de Magistris, V. G. Vaccaro, L. Verolino, "Bunched Beams in Axisymmetric Systems", submitted to: Il Nuovo Cimento.

[5] C. Chan, R. A. Jameson, Phys. Rev. E, vol. 52, p. 3074, 1995.

[6] Contributions to the 8th ICFA Advanced Beam Dynamics Workshop, Bloomington, USA, Oct. 1195, AIP conf. proc. 377, ed. S. Y. Lee. 